\title{
Morphology and Primary Crystal Structure of a Silk-Like Protein Polymer Synthesized by Genetically Engineered Escherichia coli Bacteria
}

\author{
J. PHILIP ANDERSON, ' JOSFPH CAPPELIO, ${ }^{2}$ and DAVID C. MARTIN ${ }^{1, *}$ \\ 'Macromolecular Science and Engineering Center, H. H. Dow Building, College of Engineering, The University of \\ Michigan, Ann Arbor, Michigan 48109-2136; and 2Protein Polymer Technologies, Inc., 10655 Sorrento Valley Road, \\ San Diego, California 92121
}

\begin{abstract}
SYNOPSIS
'The morphology and primary crystal structure of SLPF, a protein polymer produced by genetically engineered Escherichia coli bacteria, were characterized. SLPF is a segmented copolymer consisting of amino acid sequence blocks modeled on the crystalline segments of silk fibroin and the cell attachment domain of human fibronectin. Wide angle $x$-ray scattering (WAXS), transmission electron microscopy ( TEM), selected area electron diffraction (SAED), and molecular simulations were used to analyze the primary crystal structure of SI.PF. TFM experiments conducted on SI.PF droplets cast from formic acid on amorphous carbon film demonstrated that these protein films have a microstructure formed of woven sheaves. The sheaves are composed of well-defined whisker crystallites. The width of the whiskers, $11.8 \pm 2.2 \mathrm{~nm}$, may be correlated to the length of the silklike segment in SLPF as predicted by molecular simulations. WAXS data, TEM images, SAED, patterns, molecular simulations, and theoretical diffraction patterns all were consistent with the crankshaft model proposed for Silk I by Lotz and Keith. (c) 1994 John Wiley \& Sons, Inc.
\end{abstract}

\section{INTRODUCTION}

The biological synthesis of novel macromolecules has the potential to revolutionize the field of biomaterials design through precise control of chain composition and sequence. Via recombinant DNA technology, designed protein polymers can be produced incorporating physical, chemical, and biological properties modeled after segments of existing natural proteins. One such protein polymer is SLPF ( silk-like polymer with fibronectin cell attachment functionality). The amino acid sequence of SLPF is shown in Figure 1. SLPF can be used to coat artificial surfaces like polystyrene culture dishes to promote both the adhesion and spreading of cells. ${ }^{1,2}$ Independent films of SLPF can also be fashioned yielding cohesive protein substrates to which cells

Biopolymers. Vol. 34, 1049-1057 (1994)

(c) 1994 John Wiley \&

* To whom correspondence should be addressed have a high attachment affinity (J. Cappello, unpublished data, 1991).

The biological activity of SLPF is generated by a block of amino acids incorporating the cell attachment ligand sequence of human fibronectin. ${ }^{3}$ This sequence is recognized and specifically bound by cellular receptors known as integrins that serve to anchor cells to the extracellular matrices (ECM) in the body that contain fibronectin and other ECM proteins ${ }^{4}$ Unlike fibronectin, SLPF is a highly stable protein that resists denaturing treatments such as autoclaving, exposure to harsh solvents, absorption onto synthetic surfaces, and long-term storage at room temperature ( $\mathrm{J}$. Cappello, unpublished data, 1992)..$^{6}$

The design of the SLPF segmented copolymer combines the physical and chemical stability of silk fibroin, and the cell attachment bioactivity of fibronectin, two properties not found together in a single natural protein. The idea behind the design of the SLPF molecule was to produce chain-folded crystals with fibronectin functionalized surfaces. To fully 
$\{$ HEAD $\}-\{\text { Silk-Like }\}_{9}$ Fibronectin Segment $\}_{12}$ - $\{$ TALL $\}$

$=$

[(fM)DPVVLQRRDWENPGVTQLNRLAAHPPFASDPMGAGSGGAGAGS) GAAVTGRG IDSPASAAGY) - [(GAGAGS),GAAVTGRGDSPASAAGY $]_{12-}$

[(GAGAGS) GAGAMDPGRYQLSAGRYHYQLVWCQK |

Figure 1. The amino acid sequence of SLPF, silk-like protein polymer with fibronectin attachment functionality.

optimize performance, it is of interest to determine the influence of crystal structure and morphology on the properties of SLPF.

Previous studies have shown evidence for crystallinity in silk-like protein polymers by $\mathrm{x}$-ray diffraction. ${ }^{5}$ However, a detailed structural model that accounts for the physical properties and biological activity of SLPF was needed. Such a model could answer questions regarding the basis for SLPF's durability, rapid renaturation after exposure to harsh solvents, and ability to present cell attachment sites in a highly accessible manner while binding to synthetic, often hydrophobic surfaces. This report focuses on the crystal structure of the silk-like portion of SLPF [(GAGAGS $)_{9}$ ], which is repeated 12 times per polymer as shown in Figure 1.

The crystalline structure of natural silk has been explored by $\mathrm{x}$-ray diffraction. ${ }^{6,7}$ In an attempt to gain more information on the crystallization process, the silk producing glands from Bombyx mori were air dried and their contents were found to have a noticeably different crystal structure from the fibrous silk normally extruded from the animal. ${ }^{8}$ After further analysis, the unextruded protein structure, Silk I, was found to be easily drawn into the extruded structure, Silk II. ${ }^{9}$

Crystal structure models for Silk I are critically analyzed elsewhere. ${ }^{10}$ Proposed models have been based on hydrogen-bonded sheet type structures or helical forms. Diffraction methods for Silk I structural analysis have proven to be the most generally accepted and reproducible. The crankshaft model proposed by Lotz and Keith accounts for many aspects of the published data well. ${ }^{13}$

Lotz and Keith further the sequential model of silk fibroin crystallites, poly (AGAGSG), with synthetic poly $(A G)$. Poly ( $A G$ ) was found to be polymorphic and to have a crystal form, poly (AG) II, which is isomorphous with Silk I. From transmission electron microscopy (TFM), selected area electron diffraction (SAED), and $x$-ray powder diffraction experiments, an orthorhombic unit cell was proposed for Silk I based on poly (AG) II with space group $\mathrm{P} 22_{1} 2_{1}(a=0.47 \mathrm{~nm}, b=1.44 \mathrm{~nm}, c=0.96 \mathrm{~nm})$. The Lotz and Keith model for Silk $I$ is a compact sheet form with the polypeptide backbone in a crankshaft conformation. Hydrogen-bonded sheets can be formed from two similar chain structures. Chain I specifies the sequence poly $\left(\beta \mathrm{A}-\alpha_{\mathrm{L}} \mathrm{G}\right)$ while chain II is poly $\left(\beta A-\alpha_{D} G\right)$.

The antiparallel crankshaft model proposed by Lotz and Keith is based on regularly alternating chains I and II forming hydrogen-bonded sheets. As in the extended sheet forms, sheets stack to fill space. The face of the resulting solid, perpendicular to the chains, is thought to be composed of regular hairpin turns, where the chains switch type and direction, then proceed back through the crystal.

Diffraction data was consistent with the antiparallel crankshaft model except that certain $(0 k l)$ reflections were too intense, particularly on the first and second layer lines. Lotz and Keith proposed that this difficulty could be removed by using a model consisting of a hybrid structure in which there are two possible [001] axis offsets between neighbor sheets. The relative [001] axis positions between neighbor sheets are described by translations of zero [001] (no translation) and plus half of the $c$ axis length [ for poly(AG)]. ${ }^{12}$ Randomly alternating sheet to sheet stacking partially disrupts the periodicities creating the anomalous reflection intensities.

\section{EXPERIMENTAL}

SLPF (batch S4) was supplied by Protein Polymer Technologies, Inc., in powder form (ProNectin F). The theoretical sequence, predicted genetically by the synthetic nucleotide sequence that encodes SLPF, is shown in Figure 1 and corresponds to a molecular weight of $73 \mathrm{k}$ daltons. The protein polymer was produced in genetically engineered Escherichia coli cells, then purified from other cellular components by a process involving the extraction of the lysate with $5 M \mathrm{LiBr}$ solution. The protein was precipitated using ammonium sulfate, extensively washed, and dialyzed against deionized water. The insoluble SLPF suspension was then lyophilized to a dry powder.

The SLPF preparation was analyzed to determine its amino acid composition and elemental composition. Experimental molecular weight was determined by sodium dodecylsulfate poly (acrylamide) gel electrophoresis (SDS-PAGE). SLPF is primarily composed of the amino acids glycine $(41.5 \%)$, alanine $(31.0 \%)$, and serine $(14.8 \%)$ in a ratio of 2.83 $: 2.02: 1.00$. It was estimated from an amino acid composition analysis that approximately $93.6 \%$ of the protein contained in the $\mathrm{S} 4$ batch was SLPF. 
The elemental composition was $43.8 \%$ carbon, $18.1 \%$ nitrogen, and $6.0 \%$ hydrogen. The water content of the lyophilized powder was $5.5 \%$ and noncombustible residue was $0.6 \%$ by weight. From these results it can be concluded that approximately $94 \%$ of dry weight is organic material and based on the nitrogen value $91 \%$ is protein. The total SLPF content of the $\mathrm{S} 4$ batch is estimated to be $85 \%$.

By SDS-PAGE, the $\mathbf{S} 4$ batch consists primarily of a protein band that migrates with an apparent molecular weight of approximately $95 \mathrm{k}$ daltons. Silklike protein polymers have been shown to migrate with greater apparent molecular weights in this system when compared to soluble protein molecular weight standards (J. Cappello, unpublished data, 1992 ). Other minor bands with molecular weights smaller than $40 \mathrm{k}$ daltons, constituting less than $2 \%$ of the protein, are visible by silver staining.

\section{WAXS (Wide Angle X-Ray Scattering)}

A Rigaku $\theta-\theta$ x-ray system was used to perform WAXS on dry SLPF powder. This instrument is equipped with a $1.5 \mathrm{~kW} \mathrm{Cu} \mathrm{K \alpha}$ tube that emits $\mathrm{x}$ ray radiation at a wavelength of $0.154 \mathrm{~nm}$, and has a graphite monochromator and a $\mathrm{Nal}$ scintillation type detector. Detailed WAXS runs were conducted at $0.5^{\circ}$ per minute with sampling at $0.05^{\circ}$ intervals. Pertinent WAXS data was obtained with $0.5 \mathrm{~mm}$ etched glass substrates as sample holders between $3^{\circ}$ and $40^{\circ} 2 \theta$.

\section{Molecular Simulations}

Molecular simulations on the silk-like segment of SLPF were generated on a Silicon Graphics Personal Iris 4D25G running PolyGraf 3.0 and Cerius 3.0 (Molecular Simulations, Inc.). The force field used in PolyGraf for energy minimizations was Dreiding II. ${ }^{13}$ Energy minimizations were conducted using the Fletcher-Powell algorithm in periodic boundary conditions using the lattice sum method and allowed to converge at a maximum step of 1.5 , gradient convergence criterion of 0.1 and a minimum delta energy of $0.001 \mathrm{kcal} / \mathrm{mole}^{14}$ Crankshaft models for Silk I were minimized in PolyGraf with constrained unit cells and their diffraction patterns were calculated in Cerius. Theoretical diffraction patterns were calculated with no anomalous dispersion factor, a polarization fraction of 0.5 and no Debye-Waller factors.

Atomic coordinates for poly (AG) in the chain I crankshaft conformation from Lotz and Keith were input into Cerius. Chain II was generated from the coordinates provided for chain I by performing a mirror plane transformation along the [001] axis and then switching the positions of the carbonyl and amino groups. After transferring the atomic coordinates to PolyGraf, the corresponding molecular model of sheets composed by regularly alternating chains I and II was generated by properly binding the atoms and then minimizing the energy to convergence.

A model that is more consistent with experimental diffraction data incorporates a hybrid crystal structure and requires the second form of the crankshaft model for Silk I to be developed. The primary form has a neighbor sheet translation along the [001] axis of zero (base model) while the second form has a neighbor sheet translation of plus half the [001] axis distance (shifted model). The shifted form was also generated in Cerius and allowed to minimize in PolyGraf. Theoretical WAXS and SAED patterns were calculated for both crankshaft forms.

The Lotz and Keith crankshaft model for Silk I, poly (AG), was then fitted with serines to give poly (GAGAGS) and energy minimized within PolyGraf. Theoretical x-ray patterns based on this model were generated using Cerius. By overlaying the theoretical diffraction patterns on the experimental WAXS data, an experimental unit cell was produced by indexing the reflections and then adjusting the simulation unit cell dimensions. The crankshaft model for poly(GAGAGS) was again minimized in PolyGraf while constrained to fit within the experimental orthorhombic unit cell. $\mathrm{Fi}_{\mathrm{i}}$ nal theoretical $x$-ray diffraction patterns were calculated with effective crystallite size parameters as determined from the experimental WAXS data.

\section{TEM}

TEM sample preparation began with carbon coating freshly cleaved mica sheets to form an amorphous film in a Denton evaporator. Formic acid ( $88 \%$ ) solutions containing $1 \mathrm{mg} / \mathrm{mL}$ SLPF were atomized and sprayed onto the carbon coated mica in a hood at room temperature, covering the carbon film with solution droplets. Upon solvent evaporation, which occurred in seconds, crystallized droplets of SL.PF were left on the surface of the carbon film. This film was then floated off the mica onto reagent grade water. Four hundred mesh copper TEM grids were lifted with tweezers out of the water catching the carbon film supporting SLPF droplets.

For conventional TEM, samples were analyzed with a Phillips EM 420 operating at $120 \mathrm{kV}$. The 
sample holder was cooled with liquid nitrogen to reduce contamination. A variety of magnifications were used, typically $3 \mathrm{kX}$ to $20 \mathrm{kX}$. SAED patterns were generated from SLPF droplet samples prepared as above and then shadowed with gold in a Denton evaporator at an incident angle of about $15^{\circ}$. TEM images and SAED patterns were taken-before and after the SLPF droplets had received a critical dose of radiation. A Joyce-Loebl 3CS microdensitometer was used in conjunction with a desktop scanner, Macintosh Quadra computer, and scanner software to analyze electron diffraction data. A JEOL 2000 FX was used to estimate the total end point dose of SLPF at $200 \mathrm{kV}$.

\section{RESULTS}

\section{WAXS}

The WAXS diffractometer data from dry SLPF as received was found to have approximately eight discernible peaks as seen in Figure 2. Table I compares dry SLPF experimental data with that of the original crankshaft model $^{11}$ and also shows crystallite di-

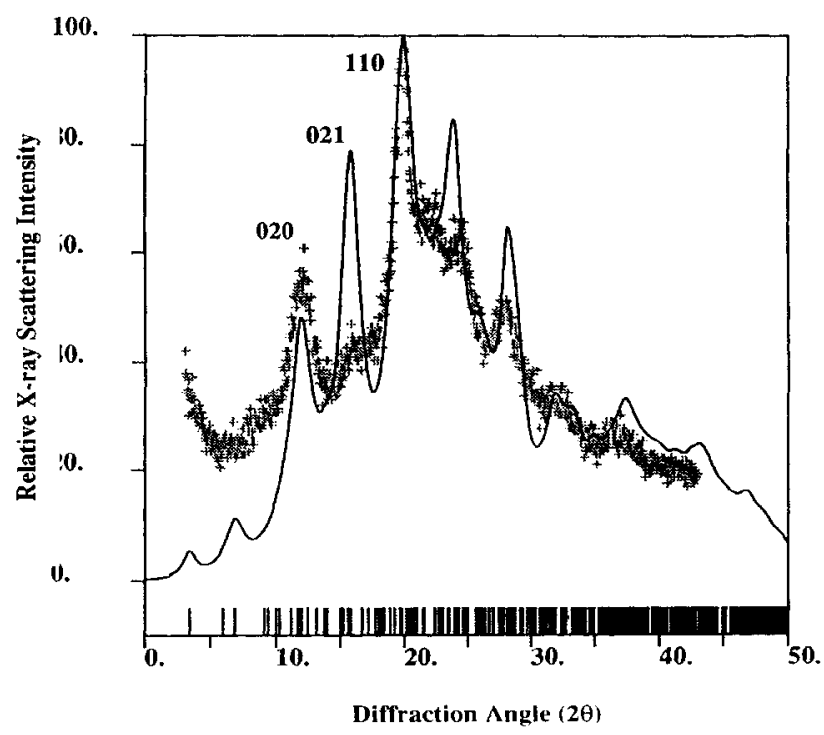

Figure 2. WAXS data from dry SLPF (crosses) with spacing at $0.741,0.558,0.451,0.409,0.370$, and $0.319 \mathrm{~nm}$. The theoretical WAXS pattern from the base poly(GAGAGS) crankshaft model (line). Note that the intensity of the theoretical (021) reflection is greater than observed as predicted by Lotz and Keith. This discrepancy may be accounted for by assuming random base and shifted sheet to sheet packing in SLPF crystals. mensions from a Sherrer analysis of the full width at half maximum of certain reflections. Reflections are indexed according to the precedent set by Lotz and Keith for poly (AG) II. The (020) reflection at $11.7^{\circ} 2 \theta(d=0.74 \mathrm{~nm})$ was calculated to arise from a crystallite size of $3.2 \mathrm{~nm}$ in the unit cell $b$ axis direction. The $(110)$ reflection at $19.7^{\circ} 2 \theta(d=0.45$ $\mathrm{nm}$ ) yields a larger crystallite size, $5.1 \mathrm{~nm}$. The effective crystallite size for ( h00) planes was estimated to be $6.1 \mathrm{~nm}$. No specific reflections could be associated with the fibronectin portion of SLPF.

\section{Molecular Simulations}

Comparison of the theoretical $x$-ray pattern with the published reflections showed the minimization of the base poly ( $\mathrm{AG}$ ) crankshaft model by Dreiding II did not give any significant deviation from the structure proposed by Lotz and Keith. Therefore it seemed reasonable to simulate the silk-like portion of the segmented copolymer, SLPF, by substituting every third alanine with serine.

The crankshaft base and shifted models for poly (GAGAGS) were both allowed to minimize under Dreiding II. Table II provides energy decompositions for shifted and base poly(GAGAGS) crankshaft models. The calculated $x$-ray diffraction data for the base poly ( GAGAGS) crankshaft model corresponds well to the experimental data as seen in Figure 2. However as predicted by Lotz and Keith, the calculated relative intensity of the (021) reflection is much greater than that of the experimental data.

This discrepancy was dealt with by employing a more realistic model which consists of regularly alternating the base and shifted sheet to sheet packing, shown in Figure 3. Shifted stacking incorporates a neighbor sheet offset of approximately $0.5 \mathrm{~nm}$ from base stacking. While the calculated (021) intensity from this hybrid model is consistent with experimental data, other periodicities arise from the regular alternation of base and shifted sheet stacking. A full Lotz and Keith model would be presumed to have both random base and shifted sheet packing within the same crystal.

Using Cerius in conjunction with dry powder WAXS data an orthorhombic unit cell for the silk fibroin portion of SLPF was experimentally deter$\operatorname{mined}(a=0.47 \mathrm{~nm}, b=1.48 \mathrm{~nm}$, and $c=0.86 \mathrm{~nm})$ for poly ( $A G$ ). Both the base and shifted models were constrained to fit within this unit cell and again minimized within PolyGraf. Effective crystallite sizes that were measured and those estimated, $8 \mathrm{~nm}$ 
Table I Comparison of Spacings and Intensities Between Poly(AG) II Data Used to Formulate the Crankshaft Model for Silk I1,9 $^{11,9}$

\begin{tabular}{|c|c|c|c|c|c|c|c|}
\hline \multicolumn{3}{|c|}{ Poly(AG) II WAXS } & \multicolumn{3}{|c|}{ SLPF Dry Powder WAXS } & \multicolumn{2}{|c|}{$\begin{array}{c}\text { SLPF Electron } \\
\text { Diffraction }\end{array}$} \\
\hline $\begin{array}{l}\text { Spacing } \\
\text { (nm) }\end{array}$ & $\begin{array}{l}\text { Relative } \\
\text { Intensity }\end{array}$ & Plane & Spacing & $\begin{array}{l}\text { Relative } \\
\text { Intensity }\end{array}$ & $\begin{array}{l}\text { Size } \\
(\mathrm{nm})\end{array}$ & Spacing & $\begin{array}{l}\text { Relative } \\
\text { Intensity }\end{array}$ \\
\hline 0.72 & 60 & $(020)$ & 0.74 & 60 & 3.2 & 0.73 & 70 \\
\hline 0.57 & 14 & $(021)$ & 0.56 & 40 & & 0.55 & 50 \\
\hline 0.45 & 100 & $(110)$ & 0.45 & 100 & 5.1 & 0.45 & 100 \\
\hline 0.39 & 18 & $(120)$ & 0.41 & 55 & & 0.39 & 52 \\
\hline 0.00 & 40 & (U4U) & 0.01 & uv & & 0.00 & $\angle 0$ \\
\hline 0.32 & 30 & (013) & 0.32 & 40 & & 0.32 & 28 \\
\hline
\end{tabular}

${ }^{a}$ WAXS data from dry SLPF powder and electron diffraction data from the whisker crystallites observed with TEM. Relevant crystallite sizes were calculated.

for (001) by best fit to WAXS data, were incorporated into Cerius to calculate the final theoretical diffraction pattern for the base model, shown in Figure 2.

\section{TEM}

Generally, SLPF droplets did not appear as uniformly dense as those formed from other polymers like poly (diacetylene) or poly(imides). ${ }^{15,16}$ The SLPF droplet morphology was not as ordered as that of poly (diacetylene) droplets. The microstructure observed resembled intertwining sheaves. These sheaves appear randomly oriented. Sheaves are

Table II Energy Decompositions of Crankshaft Models for Silk I Modified to Poly(GAGAGS) Using PolyGraf with the Dreiding II Force Field in Periodic Boundary Conditions ${ }^{a}$

\begin{tabular}{lrr}
\hline $\begin{array}{c}\text { Crankshaft Silk I Models } \\
\text { for Poly(GAGAGS) }\end{array}$ & $\begin{array}{c}\text { Base } \\
\text { Model }\end{array}$ & $\begin{array}{r}\text { Shifted } \\
\text { Model }\end{array}$ \\
\hline Total energy & -171.86 & -185.08 \\
(kcal/mole/unit cell) & & \\
(four chains per unit cell) & & \\
Bonds & 25.24 & 24.84 \\
Angles & 57.11 & 62.76 \\
Torsions & 68.68 & 55.31 \\
Inversions & $\mathbf{1 . 4 4}$ & 1.17 \\
van der Waals & 73.74 & 61.75 \\
Hydrogen bonds & -398.01 & -390.91 \\
\hline
\end{tabular}

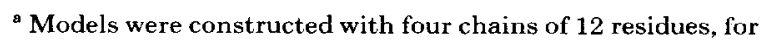
a total of 48 residues, per unit cell. formed of whisker crystallites lying parallel to one another, composing the SLPF nanostructure.

Figure 4 is part of an extended droplet showing more complete crystallization, expressed in the sheaf pattern. When more solution is sprayed on the carbon film, the droplets produced were no longer round but rather elliptical. Figure 4, part of an elliptical droplet, shows the incorporation of free whiskers on one side. Near the droplet edges one can see that the sheaves are composed of whisker crystallites. Similar single crystals of natural silk fibroin have been observed with the Silk I and II structures (Refs. 17 and 18; J. Magoshi, unpublished data, 1993).

From the analysis of 250 whiskers as shown in Figure 5, it was found that the width of these crystallites is $11.8 \pm 2.2 \mathrm{~nm}$. This experimental figure is approximately equal to the distance for the (GAGAGS $)_{9}$ repeat, in the crankshaft form, $11.6 \mathrm{~nm}$. The lengths of whiskers were up to $200 \mathrm{~nm}$, yielding aspect ratios of up to 20 .

SAED from an SLPF extended droplet shadowed with gold is shown in Figure 6 , before and after receiving the critical dose for radiation damage. Notice the disappearance of the crystalline halos immediately surrounding the central illumination. A microdensitometer analysis allowed for less obvious peaks to be identified in the SAED data and the measurement of relative intensities, as seen in Figure 7. The SAED patterns were calibrated with the gold (111) reflection and SLPF spacings are listed in Table I. The TEPD was calculated to be approximately $0.02 \mathrm{C} / \mathrm{cm}^{2}$ by estimating the time for the most outstanding diffraction ring to vanish to be 4.5 seconds at $15 \mathrm{k}$ magnification with a current density of 21 $\mathrm{pA} / \mathrm{cm}^{2}$ while operating at an accelerating voltage of $200 \mathrm{kV}$. 


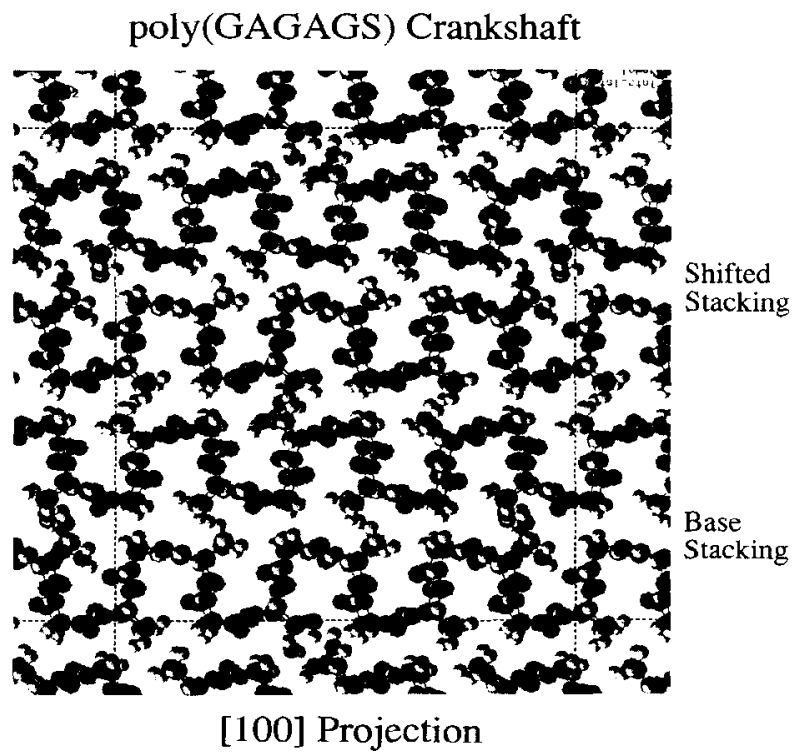

(a)

$$
\text { poly(GAGAGS) Crankshaft }
$$

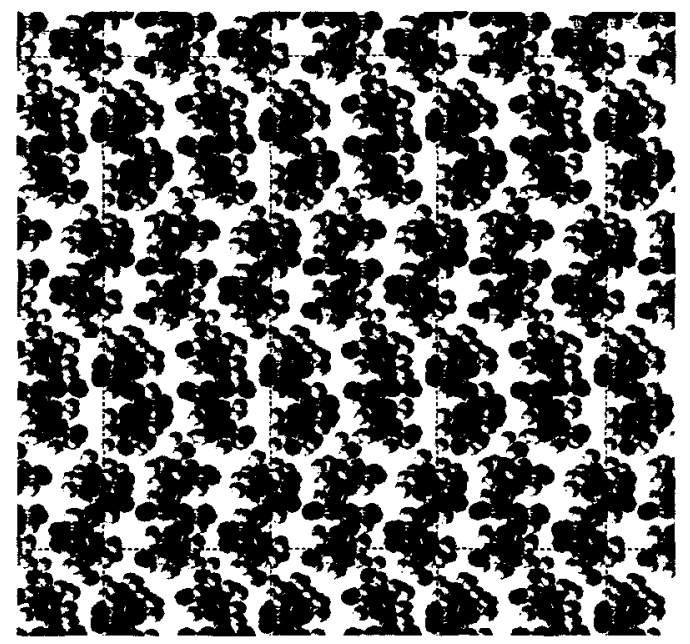

[001] Projection

(b)

Figure 3. (a) [100] projection of the regularly alternating base and shifted stacking poly (GAGAGS) crankshaft model. (b) [001] projection of the regularly alternating base and shifted stacking poly (GAGAGS) crankshaft model.

\section{DISCUSSION}

A critical comparison of SLPF dry powder WAXS data and the Lotz and Keith crankshaft model, shows good agreement (Table I). The theoretical $x$-ray diffraction pattern from the base poly(GAGAGS) crankshaft model is supported by SLPF WAXS data, as seen in Figure 2. Hence, it is concluded that the poly (GAGAGS) crankshaft model for Silk $I$ is a viable model for the SLPF crystal structure. Note that a Silk II model does not fit the data because the Silk II diffraction lacks reflections in the both the $0.7 \mathrm{~nm}$ and $0.5 \mathrm{~nm}$ ranges and includes a spacing at $0.93 \mathrm{~nm} .{ }^{19}$

As listed in Table II, energies calculated for the base crankshaft model and shifted model are similar. When the structure is actually formed the existence of the two possible neighbor sheet positions may give the crystal additional freedom in packing. Lotz and Keith proposed that such a structure would solve discrepancies between experimental and calculated diffraction patterns. Algorithms are being explored to calculate theoretical diffraction intensities for randomly alternating base and shifted sheet to sheet packing.

Table I shows the calculated effective crystallite dimensions using the Scherrer equation. The (020) reflection has a width corresponding to an effective crystallite size of $3.2 \mathrm{~nm}$ in the [010] unit cell axis direction, which is smaller than the effective crystallite size calculated from the (110) reflection, 5.1 $\mathrm{nm}$. The (110) reflection has components in both the [100] and [010] unit cell axis directions. This data further supports the proposed crankshaft model, as hydrogen bonding occurs in the crystal parallel to the [100] axis, while only van der Waals forces are present in the [010] direction. Therefore it is reasonable to expect that the effective crystallite dimensions will be larger in the [100] direction than in the [010] as observed.

The TEM data shows that the fundamental unit of morphology of SLPF is a whisker crystal. The measured width of the SLPF whisker crystallites is $11.8 \pm 2.2 \mathrm{~nm}$. This is consistent with the $11.6 \mathrm{~nm}$ width predicted for the silk-like portion of the segmented polypeptide between fibronectin segments by the poly (GAGAGS) crankshaft model. The theoretical whisker width is calculated on the basis of 27 times half of the [001] axis unit cell length of $0.86 \mathrm{~nm}$ [ the unit cell is for poly ( AG) and its length represents the distance spanned by four residues].

All whiskers have a very similar width and do not appear to grow in the proposed [001] direction. Solvent concentration gradients and variation in evaporation rate appear to effect the packing of whiskers, as they are observed to organize into films and sheaves forming both round droplets and extended structures. None of these processing variations in- 


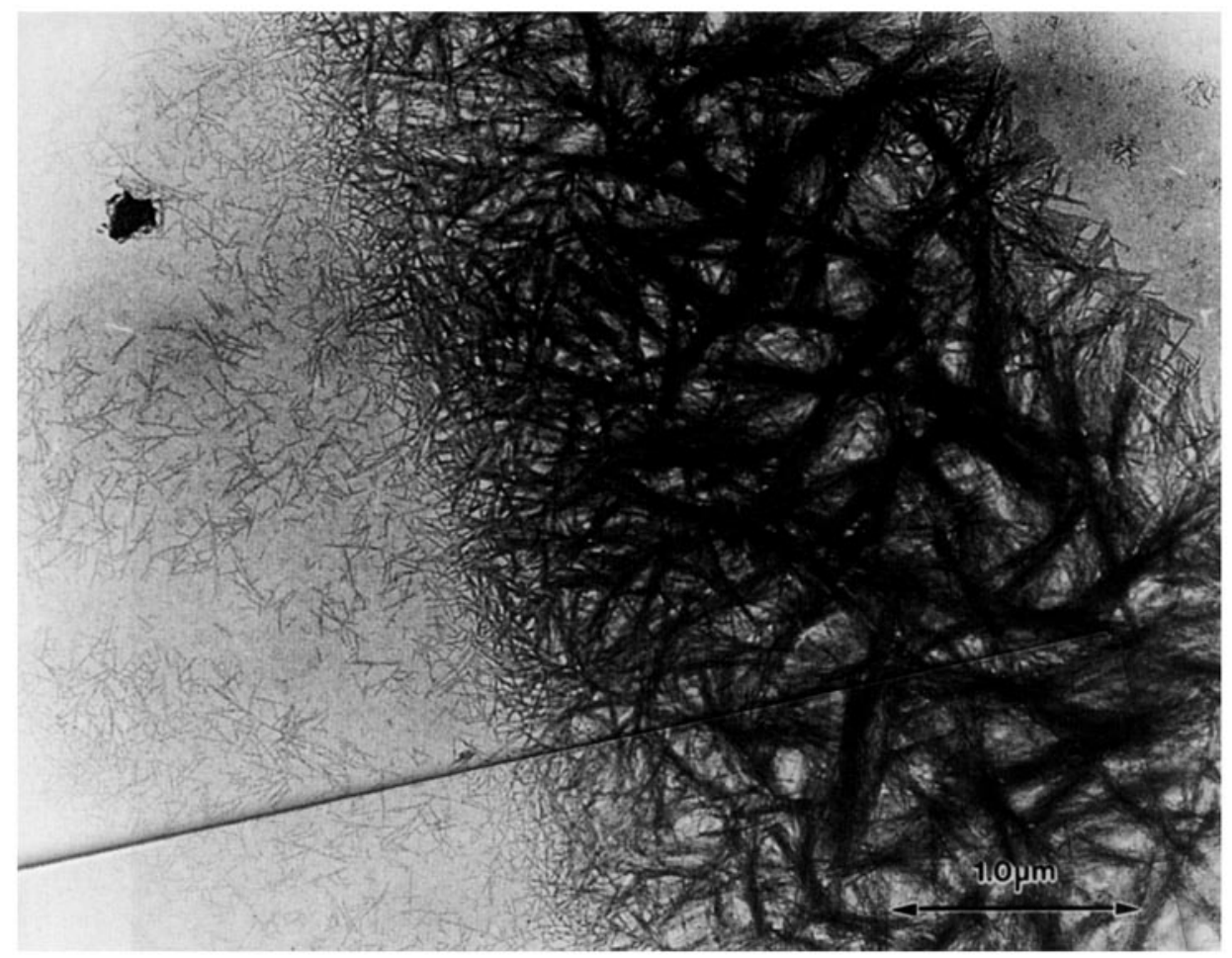

Figure 4. Extended SLPF droplet crystallized out of formic acid. Note the free whiskers on the left side of the photomicrograph.

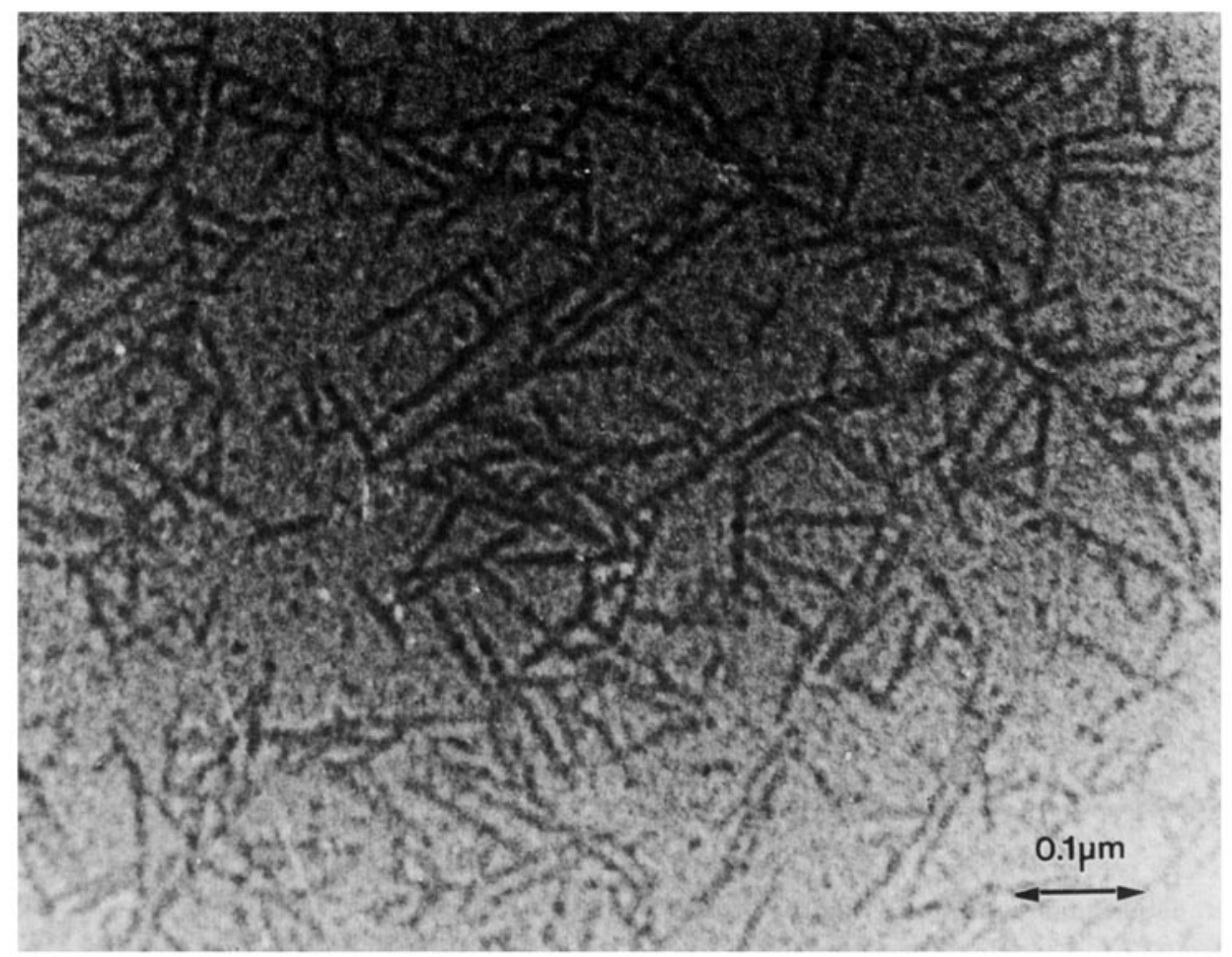

Figure 5. Free whisker film near extended SLPF droplet. The width of each whisker is $11.8 \pm 2.2 \mathrm{~nm}$ 


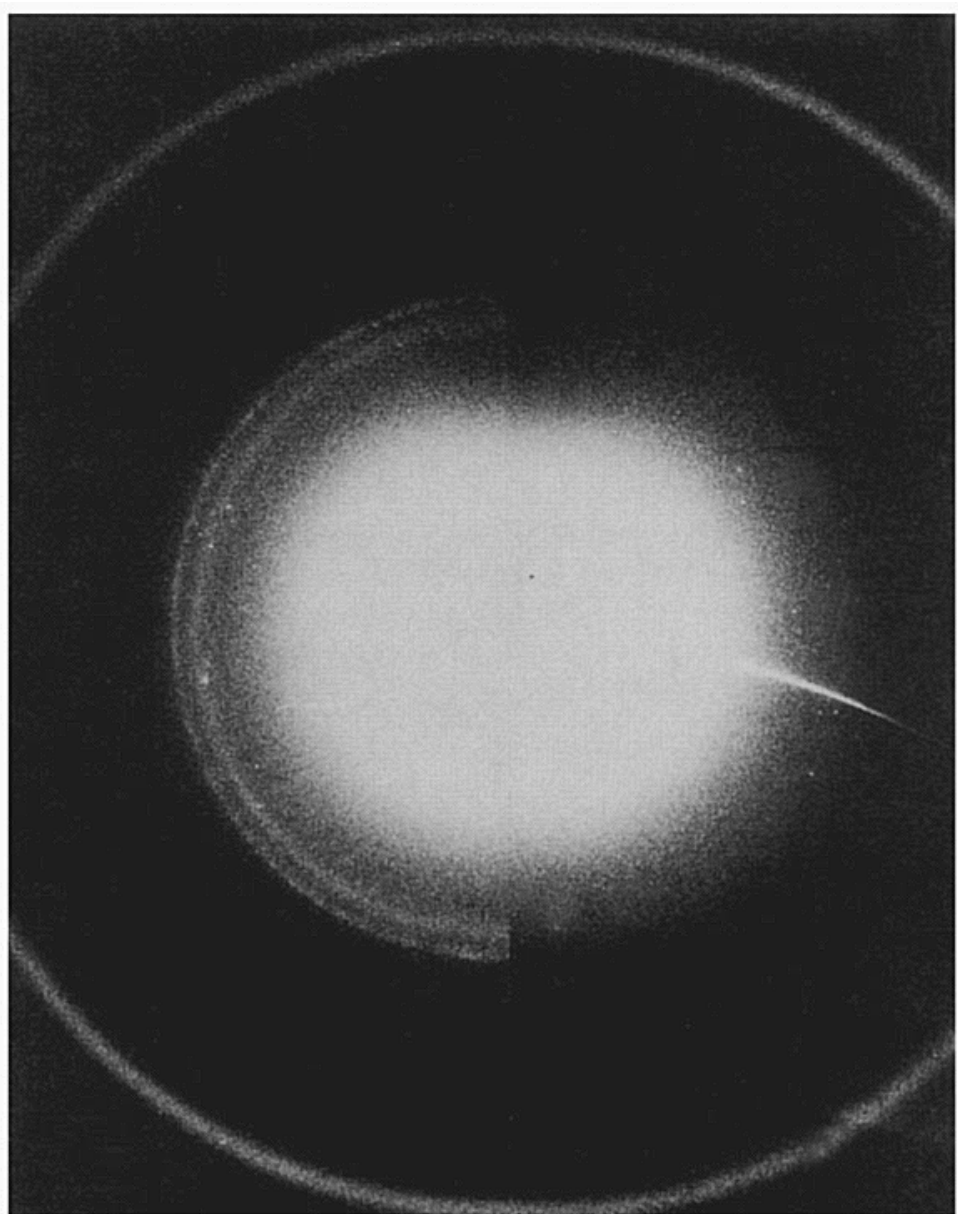

Figure 6. SAED from a gold-shadowed SLPF droplet before (left) and after (right) receiving the critical dose of radiation.

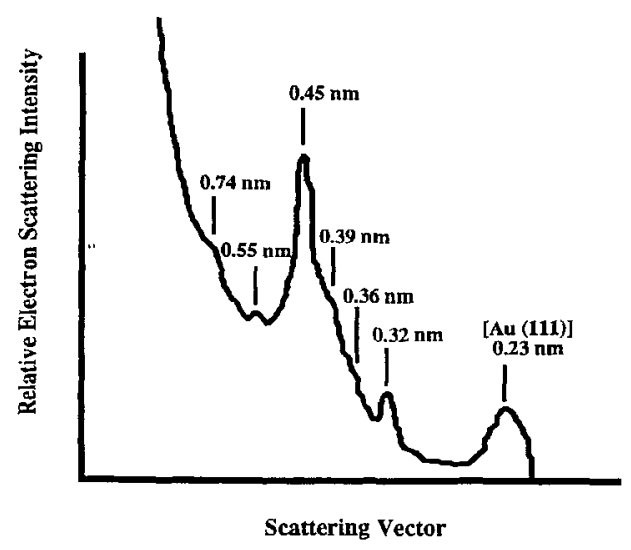

Figure 7. Microdensitometer analysis of SAED data from gold shadowed SLPF droplets. Spacings and relative intensities are consistent with the Silk I crystal structure. fuence the observed $11.6 \mathrm{~nm}$ whisker width. These observations suggest that the width of the crystallite whiskers is strictly controlled by the length of the silk-like segment. The distribution observed in whisker length is consistent with kinetically controlled nucleation and growth.

The SAED patterns also show reflections consistent with the Silk I form. If the whiskers were in the extended Silk II form spacings near 0.74 and $0.55 \mathrm{~nm}$ would not be present and the whiskers would most likely be wider than the $11.6 \mathrm{~nm} \mathrm{ob-}$ served, due to the extension in the proposed [001] unit cell dimension. Silk II reflections include spacings at $0.93 \mathrm{~nm}$ that are not observed. ${ }^{19}$ Furthermore, silk fibroin observed in the Silk II conformation has traditionally required the sample to be subjected to a shear stress or otherwise extruded. 


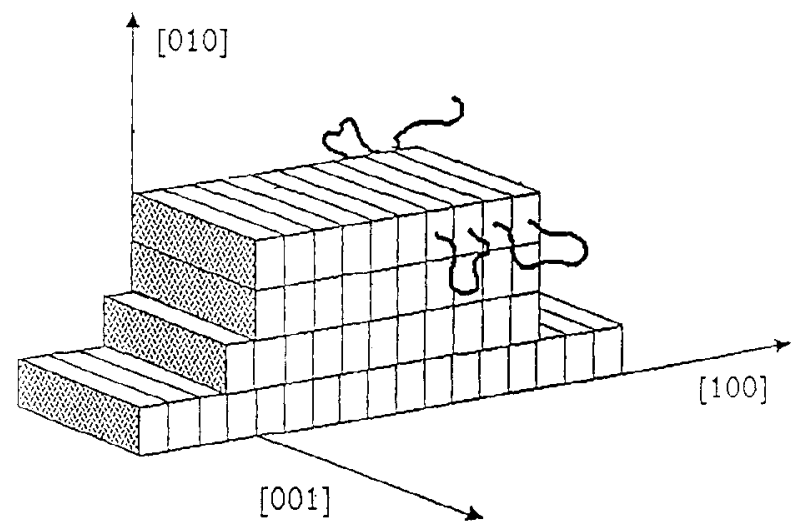

Figure 8. A schematic representation of SLPF crystal structure orientation within whiskers. Grey faces (100) have hydrogen bonding potential. Black curves represent fibronectin turns whose structures have not yet been determined.

A schematic model for an oriented SLPF whisker crystallite is presented in Figure 8. Whisker growth is apparently chemically controlled in one dimension [001] and is determined by the length of the silklike blocks composing SLPF. Hydrogen bonding is likely to be heavily promoted and thought to occur in the $[100]$ direction, parallel to both the substrate and the length of the whisker. 'The weaker van der Waals forces responsible for the stacking of sheets in the [010] direction are likely to be compromised in favor of growth in the [100] direction and thus the (010) planes are in direct contact with the substrate and also have a larger surface area.

In this sheet type structure it is thought that the sides of the whisker coincide with the fibronectin segment and then the next silk-like segment proceeds back through the crystallite in an antiparallel fashion. ${ }^{20}$ Due to the length and complexity of the fibronectin segment the ends of the sheet may not be hairpin turns. The sides of the whiskers could be disordered, perhaps even including some of the silklike sequence, forming a patch cord mesh before proceeding back into the crystal.

The physical and biological properties of SLPF are consistent with the observation of whiskers. The crystalline cores of the whiskers may lend durability and renaturation features to SLPF. The apparent structural freedom associated with the packing of the fibronectin segment of the copolymer may allow a range of possible conformations. Some of these conformations may be more biologically active than others. Perhaps superficial fibronectin segments lend themselves to accept induced fits with cellular receptors. Certainly the whisker basis of the morphology is conducive to presenting functional sequences at film surfaces. The relationship between the concentration of SLPF in formic acid and the crystallinity of films and droplets is currently being explored. ${ }^{21}$

Little definitive information about the structure of fibronectin segment of the copolymer was obtained. It does not appear that the fibronectin segment adds any discernible features to diffraction patterns. When comparing the measured width of the whisker crystallites with the predictions of the crankshaft model, it seems possible that fibronectin segments form turns composing the surface along the length of the whisker, normal to the substrate. However, the precise chain orientation within the whisker remains to be ascertained.

\section{CONCLUSIONS}

This research has shown that the biologically designed protein polymer, SLPF, the product of genetically engineered $E$. coli cells, is semicrystalline in nature and has a crankshaft crystal structure similar to that proposed by Lotz and Keith for Silk I. WAXS, SAED, molecular simulations, and theoretical diffraction information all support the assertion that crystallite whiskers are in the Silk I form. The whiskers are of varying lengths but of uniform width, $11.8 \pm 2.2 \mathrm{~nm}$. The width can be compared to the theoretical length of the silk-like portion of SLPF, the [001] axis length of nine (GAGAGS) units in the crankshaft conformation, 11.6 $\mathrm{nm}$. Given the similarity in these distances, it is possible to envision an antiparallel folding model with the [001] axis nominally perpendicular to the length of the whisker.

This work has been supported by Protein Polymer Technologies, Inc., of San Diego, CA. The authors would like to thank Jun Liao for assistance on the Phillips EM 420.

\section{REFERENCES}

1. Esty, A. (1992) Biomedical Products 16 (5), 76-78.

2. Esty, A. (1991) American Biotechnology Laboratory $\mathbf{9}(3), 44$.

3. Cappello, J. \& Crissman, J. W. (1990) Polym. Pre prints 31, 193.

4. Ruoslahti, E. \& Pierschbacher, M. D. (1987) Science 238, 491-497. 
5. Cappello, J., Crissman, J. W., Dorman, M., Mikolajczak, M., Textor, G., Marquet, M. \& Ferrari, F. A. (1990) Mat. Res. Soc. Symp. Proc. 174, 267.

6. Shimizu, M. (1941) Bull. Imp. Sericult. Expt. Sta. Jpn. 10,475 .

7. Fraser, R. D. B. \& MacRae, T. P. (1973) Conformation in Fibrous Proteins and Related Synthetic Polypeptides, Academic Press, New York, chap. 13.

8. Brill, R. (1930) Naturwiss 18, 622.

9. Kratky, O., Schauenstein, E. \& Sekora, A. (1950) Nature 165, 319-320.

10. Anderson, J. P. \& Martin, D. C. (1993) Biopolymers, submitted.

11. Lotz, B. \& Keith, H. D. (1971) J. Mol. Biol. 61, 201215.

12. Lotz, B. \& Cesari, F. C. (1979) Biochimie 61, 205214.

13. Mayo, S. L., Olafson, B. D. \& Goddard, W. A. III (1990) J. Phys. Chem. 84, 8897-8909.

14. Karasawa, N. \& Goddard, W. A. III (1989) J. Phys. Chem. 94, 7320-7327.
15. Wilson, P. W. \& Martin, D. C. (1992) J. Mat. Res. $7(11), 3150-3158$.

16. Martin, D. C., Berger, L. L. \& Gardner, K. H. (1991) Macromolecules 24, 3921-3928.

17. Lotz, B., Gonthier-Vassal, A., Brack, A. \& Magoshi, J. (1982) J. Mol. Biol. 156, 345-357.

18. Magoshi, J. (1977) Polymer 18, 643-646.

19. Fraser, R. D. B., MacRae, T. P. \& Stewart, F. H. C. (1966) J. Mol. Biol. 19, 580.

20. Parkhe, A. D., Fournier, M. J., Mason, T. L. \& Tirrell, D. A. (1993) Macromolecules 26, 6691-6693.

21. Anderson, J. P., Steven-Hassard, M. \& Martin, D. C. (1994) in Silk Polymers: Materials Science and Biotechnology, Kaplan, D., Adams, W. W., Farmer, B. \& Viney, C., Eds., American Chemical Society, Washington $\mathrm{DC}$, chap. 12.

Received September 14, 1993

Accepted February 1, 1994 